\title{
A Computer Algorithm for Determining the Hausdorff Dimension of Certain Fractals
}

\author{
By Lucy Garnett
}

\begin{abstract}
A fractal is a set which has nonintegral Hausdorff dimension. Computation of the dimension directly from the definition would be very time-consuming on a computer. However, the dimension can be computed using Newton's method if there exists a self-expanding map on the set. This technique is applied to compute the dimension of the Julia set of the quadratic mapping $z \rightarrow z^{2}+c$ for small real values of $c$.
\end{abstract}

1. Fractals and Hausdorff Dimension. B. Mandelbrot [4] introduced the word fractal as "a set for which the Hausdorff-Besicovitch dimension strictly exceeds the topological dimension." Whereas the topological dimension of any subset of the plane is either one or two, the Hausdorff dimension may be nonintegral. Physicists [7] use the word "strange" in this context.

Informally, the Hausdorff dimension of a subset $X$ of a metric space can be pictured by overlaying an $n$ by $n$ gridwork of boxes on $X$ for arbitrarily large values of $n$. In this manner, $X$ is covered by $n^{2}$ boxes. If $X$ is linear, then only $n$ of the boxes intersect $X$, and $X$ has Hausdorff dimension one. If $X$ covers most of the plane then approximately $n^{2}$ of the boxes intersect $X$, and $X$ has Hausdorff dimension two. If $X$ falls between the first and second case, then $X$ intersects $n^{d}$ boxes for some value of $d$ between one and two, and $X$ is a fractal with Hausdorff dimension $d$. In other words, if $X$ has dimension $d$, then approximately $n^{d}$ boxes of size $1 / n$ are needed to cover $X$. This might be of interest when drawing $X$ on some coarse device such as a graphics terminal, where a point on the device is in reality a ball of size $1 / n$. Strictly speaking, the "gridwork picture" describes the Minkowski dimension. It is equal to the Hausdorff dimension in all "nice" cases which includes all the sets discussed in this paper. More formally:

Definition. Let $C(\varepsilon)$ be a covering on $X$ by countably many balls $\left\{B_{i}\left(r_{i}\right)\right\}_{i \in I}$ of radius $r_{i} \leq \varepsilon$. For each real number $\sigma>0$ form the sum $\sum_{i \in I} r_{i}^{\sigma}$. Take the infimum of the sum over all coverings $C(\varepsilon)$. Define a function $H_{\sigma}$ by taking the limit of the infimums as $\varepsilon$ approaches zero. Notationally, this says

$$
H_{\sigma}(X)=\lim _{\varepsilon \rightarrow 0} \inf _{C(\varepsilon)}\left(\sum_{i \in I} r_{i}^{\sigma}\right) .
$$

This is a way of measuring the area needed to cover $X$ by small balls, with the convention that a ball of radius $r$ has area $r^{\sigma}$. If $\sigma$ is too small, then $H_{\sigma}(X)$ is infinite. If $\sigma$ is too large, then $H_{\sigma}(X)$ is zero. There is a unique value where

Received September 17, 1985; revised July 31, 1987.

1980 Mathematics Subject Classification (1985 Revision). Primary 58F21, 30D05. 
this function passes from infinite to finite. This critical number $\delta$ is the Hausdorff dimension of $X$, and $H_{\sigma}(X)$ is the Hausdorff measure of $X$ in $\sigma$.

In some sense the Hausdorff dimension gives the number of linear dimensions. See Rogers' book [8] for a fuller discussion. It is expensive and difficult to implement this definition on a computer. However, if $X$ has a self-expanding map, there is another approach derived from theorems proven by R. Bowen [1] and D. Sullivan [10].

THEOREM. Let $f: X \rightarrow X$ be an expanding complex analytic map. Then there exists a unique probability measure $\mathbf{m}$ on $X$ and a unique real number $\delta$ such that $f$ expands $\mathbf{m}$ by a factor of $\left|f^{\prime}\right|^{\delta}$. Furthermore, the Hausdorff dimension of $X$ is $\delta$ and $\mathbf{m}$ represents Hausdorff measure. That is, for any measurable subset $A$ of $X$ the following equation holds:

$$
\mathbf{m}(f(A))=\int_{z \in A}\left|f^{\prime}(z)\right|^{\delta} \mathbf{m}(d z) .
$$

Let us use this theorem to calculate the Hausdorff dimension of two well-known sets.

Example 1. Let $X$ be the entire 2-dimensional plane. Set $f$ equal to the doubling map. Namely, $f(x, y)=(2 x, 2 y)$ and $f$ satisfies the hypotheses of the theorem above. Let $\mathbf{m}$ be the usual Lebesgue measure. If $A$ is a subset of $X$, say a ball of radius $r$, then $\mathbf{m}(A)=\pi r^{2}$ and $\mathbf{m}(f(A))=\pi(2 r)^{2}=4 \pi r^{2}$. Since $f^{\prime}(z)=2$ for all values of $z$, we see that Eq. $(*)$ is satisfied for a value of $\delta$ equal to two. Uniqueness of the solution implies that the plane has Hausdorff dimension two. Although this result is by no means startling nor new, it is reassuring.

Example 2. Let $X$ be the middle-third Cantor set. If we represent the points in the unit internal $[0,1]$ as sequences of zeros, ones and twos, then the Cantor set consists of all those sequences which contain only zeros and twos. The expanding self-map $f$ is obtained by stretching the Cantor set by a factor of 3 and then laying it over itself. More precisely, if $z$ is between 0 and $1 / 3$, then $f(z)=3 z$. Otherwise, $f(z)=3 z-2$. This is a double covering with derivative constantly equal to 3 . Let $\mathbf{m}$ be the Lebesgue measure. If $A$ is a subset of $X$, then $\mathbf{m}(f(A))=2 \mathbf{m}(A)$. Equation $(*)$ is satisfied if $3^{\delta}=2$. Solving for $\delta$, we find that the Hausdorff measure of the middle-third Cantor set is $\log 2 / \log 3$.

The Hausdorff dimensions of both these examples have been known for a long time. The remainder of this paper is devoted to finding the Hausdorff dimension of the Julia set of a quadratic map. There have been no previous numerical approximations to these dimensions. Indeed, they have only recently been found to be nonintegral [10].

2. Julia Sets. Julia sets arise naturally in the study of the dynamical systems formed by iterating complex analytic maps from the plane to itself. The study of these systems was initiated in the early 1900's by P. Fatou and G. Julia. We shall restrict our attention to quadratic maps of the form $f(z)=z^{2}+c$. A major concern in the study of dynamical systems is the classification of orbits. The orbit of the point $z$ is the sequence of points $z, f(z), f(f(z)), f(f(f(z)))$, ad infinitum. A main question is: When does the orbit of a point converge to a fixed or periodic point, and when does it have no such stability but instead moves around erratically? Consider 
the example $f(z)=z^{2}$. Points inside the unit circle have orbits converging to the origin. Points outside the unit circle converge to infinity. Points on the unit circle itself (for the most part) move around the unit circle in a complicated manner. Thus the complex plane is split into two different sets. The stable points are those for which points nearby stay nearby under the action of $f$. In the above example this corresponds to those points whose magnitude is not equal to one. The unstable points are those for which nearby points spread apart. The latter set is called the Julia set of the mapping $f$. It follows that $f$ is a self-expanding map on the Julia set.

The Julia set in the above example is the unit circle. However, if the map $f$ is perturbed just slightly by adding a small constant $c$ to the $z^{2}$ term, then the Julia set becomes infinitely complicated. The first investigations of the morphology of Julia sets go back to B. Mandelbrot [5]. The set of complex values $c$ for which the Julia set is connected is called the Mandelbrot set and is represented in Figure 1 by the black set. This set is generated by using the following theorem [1]: The Julia set of the function $f(z)=z^{2}+c$ is connected if and only if the sequence of sums $c, c^{2}+c,\left(c^{2}+c\right)^{2}+c, \ldots$ does not become infinite. Figures 2 and 3 represent

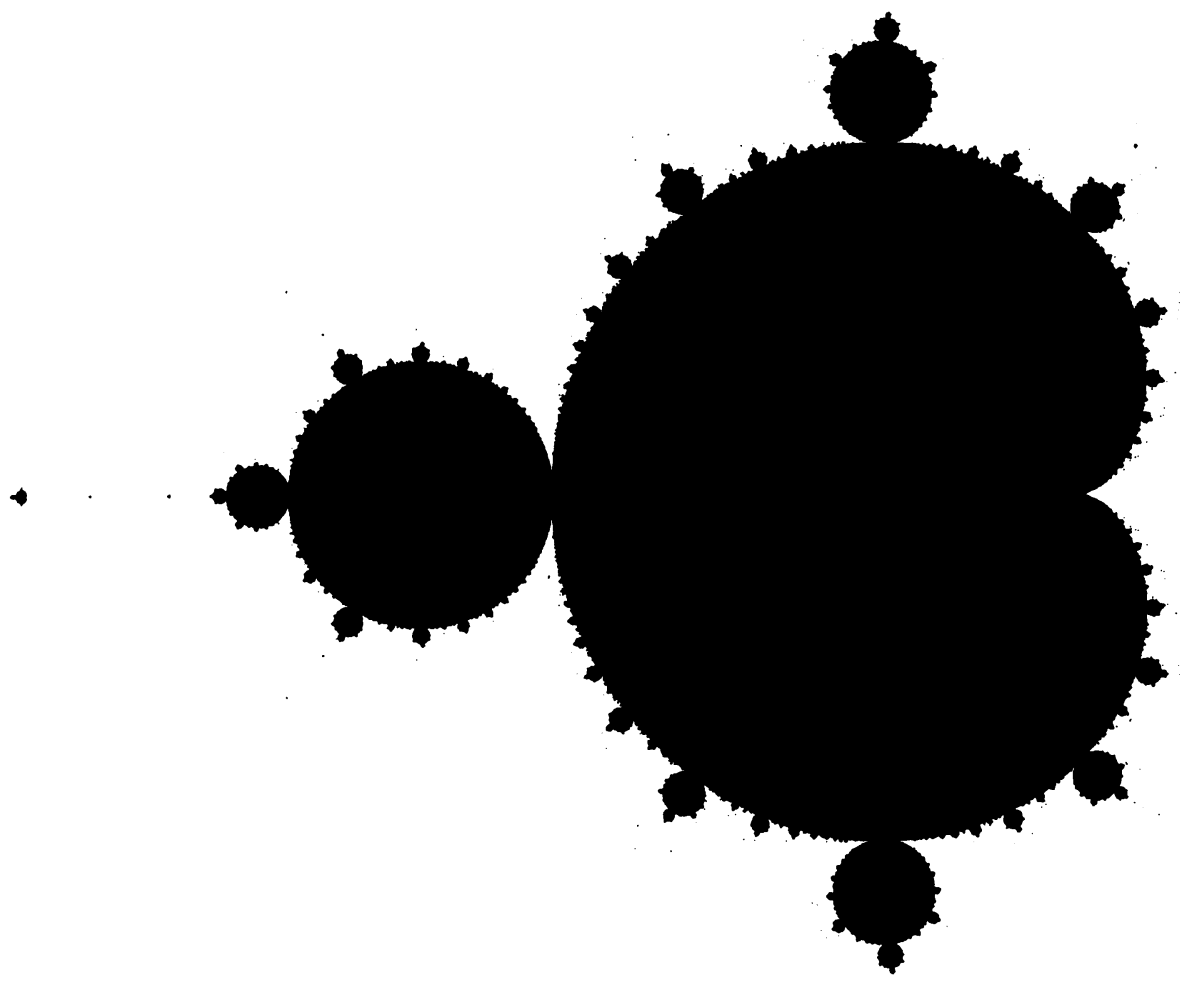

Figure 1

The Mandelbrot set is the dark area. The figure has symmetry about the real axis. The real part of the main cartoid varies from -.75 to .25 . The entire figure is contained within a box of length 4 centered at the origin. The leftmost point hits the edge of this box at $(-2,0)$. The algorithm for computing the Hausdorff dimension works only for real values of $c$ lying in the main cartoid. 


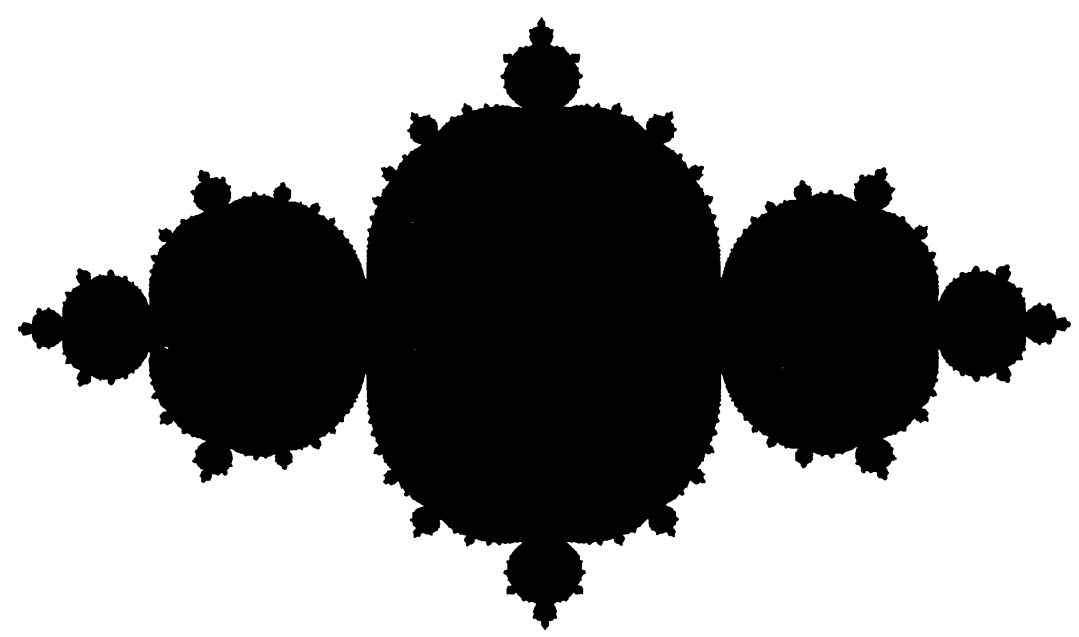

Figure 2

The boundary of the dark region is the Julia set for a real value of $c$, approximately -.6 , located near the leftmost boundary of the main cartoid of the Mandelbrot set.

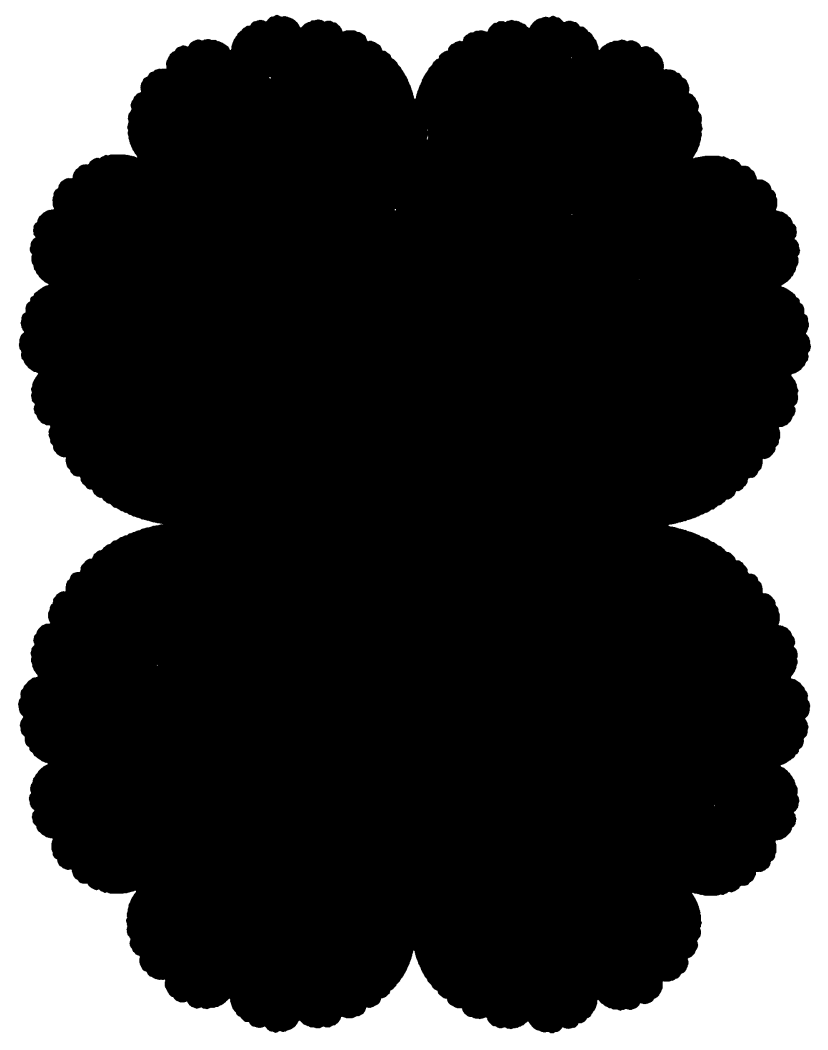

FIGURE 3

The boundary of the dark region is the Julia set for a real value of $c$, approximately .2, located near the rightmost boundary of the main cartoid of the Mandelbrot set. 


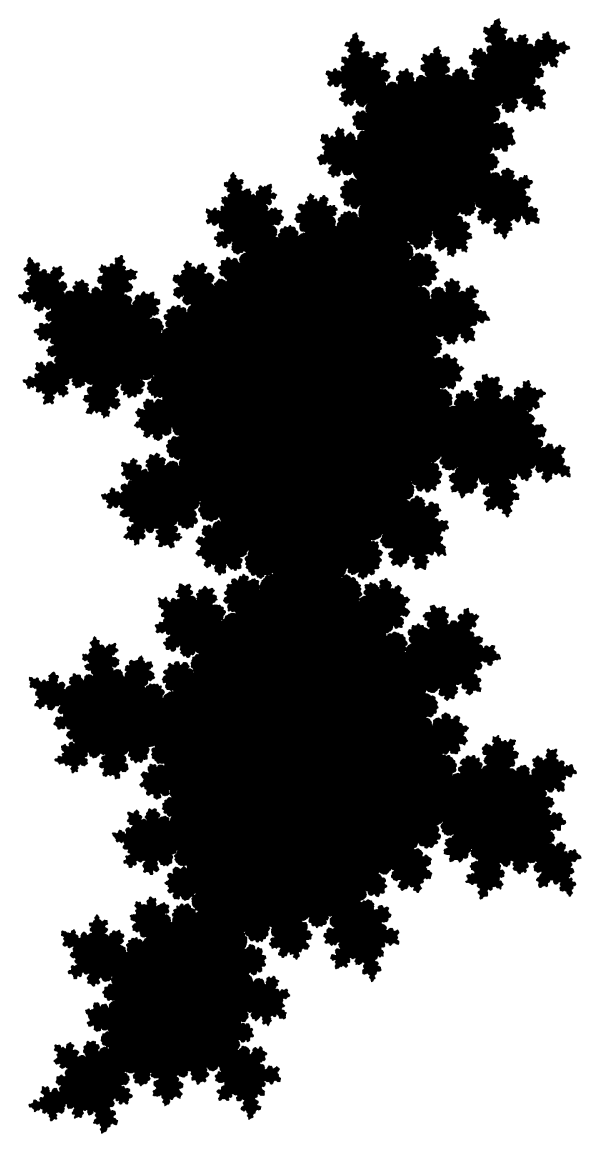

FIGURE 4

The boundary of the dark region is the Julia set for a complex value of $c \approx(-.6, .1)$. The algorithm presented in this paper does not apply to this case.

the Julia sets for two real values of $c$ occurring near the left and right boundaries of the main cartoid-like body of the Mandelbrot set. They were generated by starting with an unstable fixed point of $f$ and performing a backwards iteration. This algorithm works because the inverse orbit of any point in the Julia set is dense in the Julia set [10]. Since $f$ is expanding on the Julia set, $f^{-1}$ is contracting on the Julia set, and therefore backwards iteration is a stable procedure. Two sources of detailed color pictures are Mandelbrot's book [4] and the August 1985 issue of Scientific American. Figures 1, 2, 3 and 4 in this paper are courtesy of Dr. Alan Norton, IBM Thomas J. Watson Research Center.

The question that we investigate is: How does the Hausdorff dimension of the Julia set of $z^{2}+c$ depend upon the value of $c$ ? Attention is now restricted to those values of $c$ lying inside the main body of the Mandelbrot set. D. Ruelle [9] proved that the Hausdorff dimension is an analytic function of $c$. For $c$ equal to zero we have seen that the Julia set is the unit circle and therefore has Hausdorff dimension equal to one. D. Sullivan [10] proved that for $c$ greater than zero the Hausdorff dimension is greater than one. For the remainder of the paper we consider only real 
values of $c$ in the main body. Hence from now on $c$ is a real value lying between -.75 and +.25 . Since $f$ is a self-expanding map on the Julia set, our algorithm searches for a solution to Eq. (*). It uses Newton's method to do this. The idea for the procedure is due to $\mathrm{W}$. P. Thurston.

\section{The Algorithm.}

\section{Step 1. Finite Approximation}

We want to partition the Julia set of $f(z)=z^{2}+c$ into many very small sets in order to approximate the integral in Eq. (*) by finite sums. Choose an integer $n$. We will generate a binary tree of depth $n$ whose nodes are points on the Julia set and partition the set into $2^{n}$ pieces. First find the unique expanding fixed point of $f$. This is achieved by solving the equation $z^{2}-z+c=0$. In general there are two solutions, but the larger one is the expanding one. If we denote this solution by $w$, then $-w$ is the root of the binary tree. The rest of the tree is filled in by iterating $f$ backwards $n$ times as described in the previous section. The children of a node are the inverse image of their parent. The inverse image with smallest imaginary part is the left child. This convention arranges the points at any given level in circular order. The $n$th level will have $2^{n}$ points $z_{1}, \ldots, z_{2^{n}}$ and determines a partition of the Julia set into $2^{n}$ subsets $\left\{A_{i}\right\}_{1 \leq i \leq 2^{n}}$ centered about the points $z_{i}$.

\section{Step 2. Setting up Eq. (*)}

Since the $A_{i}$ are in circular order and $f$ is a double covering on the Julia set, we have

$$
f\left(A_{i}\right)=A_{2 i-1} \cup A_{2 i}
$$

and

$$
\mathbf{m}\left(f\left(A_{i}\right)\right)=\mathbf{m}\left(A_{2 i-1}\right)+\mathbf{m}\left(A_{2 i}\right) .
$$

$A_{i}$ is a small set with center $z_{i}$, and we approximate the derivative function $f^{\prime}(z)$ on $A_{i}$ by $f^{\prime}\left(z_{i}\right)$. Hence, a finite approximation of Eq. $(*)$ is

$$
\mathbf{m}\left(A_{2 i-1}\right)+\mathbf{m}\left(A_{2 i}\right) \approx\left|f^{\prime}\left(z_{i}\right)\right|^{\delta} \mathbf{m}\left(A_{i}\right) \text { for all } i \text {. }
$$

Rewriting this equation, we get

$$
\left(\mathbf{m}\left(A_{2 i-1}\right)+\mathbf{m}\left(A_{2 i}\right)\right) /\left|f^{\prime}\left(z_{i}\right)\right|^{\delta} \approx \mathbf{m}\left(A_{i}\right) .
$$

Equation $(* *)$ only holds when $\delta$ is the Hausdorff dimension. If we view the lefthand side of this equation as a transformation on measures, then we can restate the problem as one of searching for a fixed point. More precisely, for any $\sigma>0$ define $\mathbf{T}_{\sigma}$ to be a linear operator on measures as follows:

$$
\mathbf{T}_{\sigma}\left(\mathbf{m}\left(A_{i}\right)\right)=\left(\mathbf{m}\left(A_{2 i-1}\right)+\mathbf{m}\left(A_{2 i}\right)\right) /\left|f^{\prime}\left(z_{i}\right)\right|^{\sigma} .
$$

The problem then becomes to find a combination of $\delta$ and $\mathbf{m}$ such that $\mathbf{m}$ is a fixed point of $\mathbf{T}_{\delta}$. That is, $\mathbf{T}_{\delta}\left(\mathbf{m}\left(A_{i}\right)\right)=\mathbf{m}\left(A_{i}\right)$ for all $i$. This $\delta$ will approximate the Hausdorff dimension of $X$.

\section{Step 3. Initial Conditions}

Start the procedure with a guess of $\sigma=1$ and the uniform measure $\mathbf{m}\left(A_{i}\right)=1 / 2^{n}$ for every $i$. 


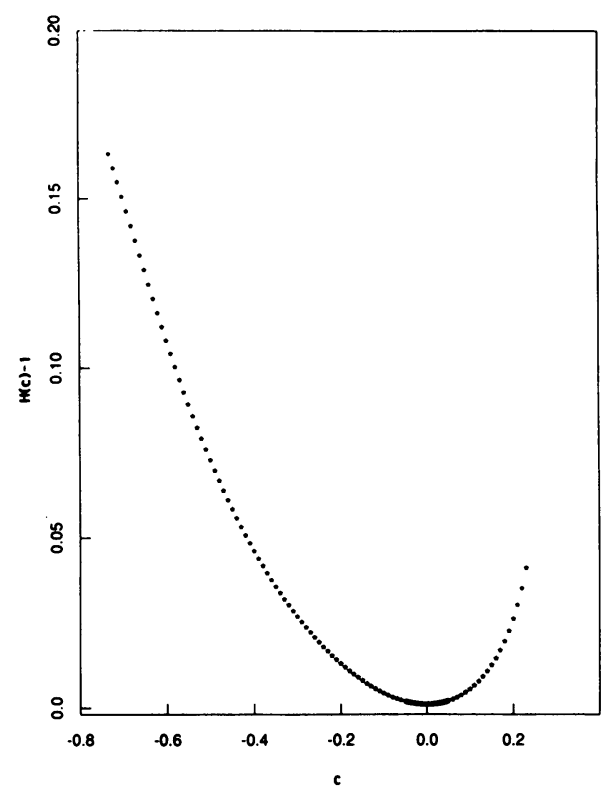

(a)

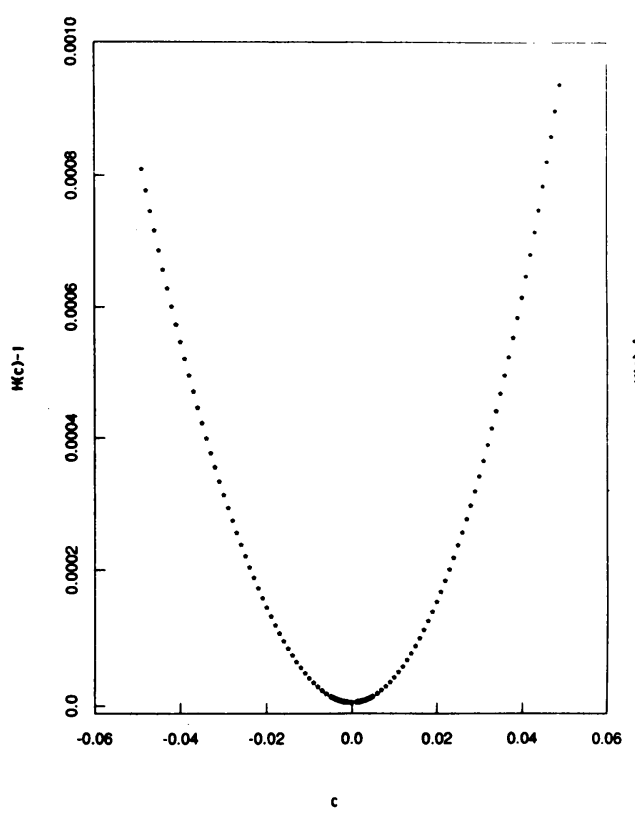

(c)

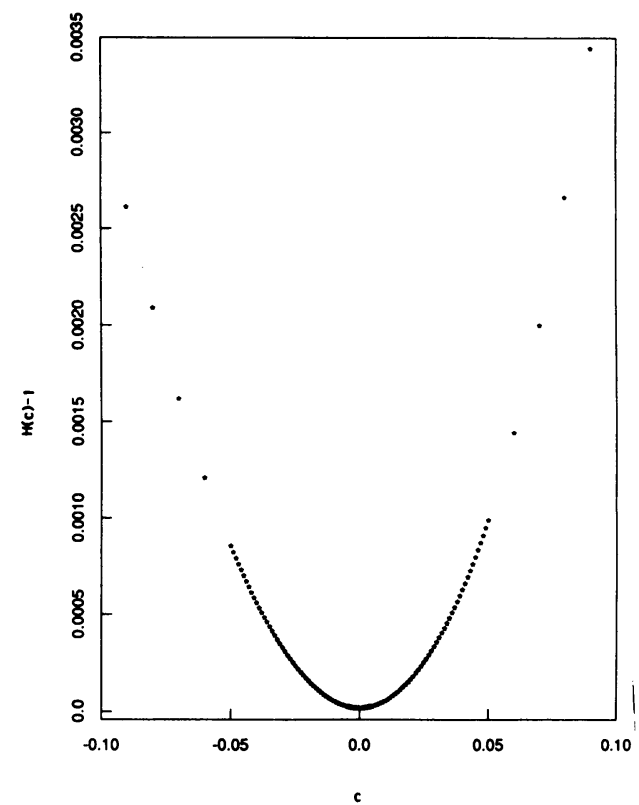

(b)

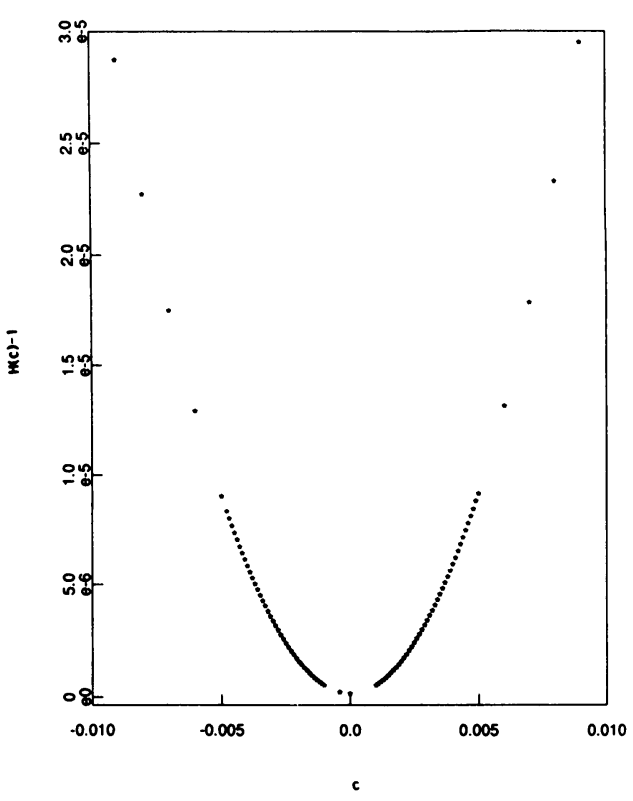

(d) 


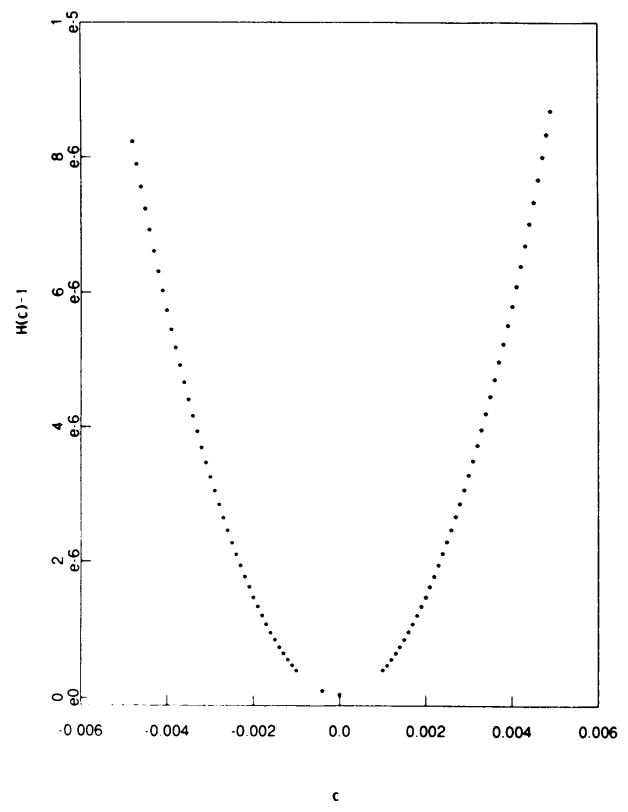

(e)

\section{FIGURES $5 \mathrm{a}-\mathrm{e}$}

$H(c)$ is the Hausdorff dimension of the Julia set generated by the mapping $z \rightarrow z^{2}+c$. Since the Julia set generated by $z \rightarrow z^{2}$ is the circle with dimension one, the graphs plot $H(c)-1$ vs $c$. Figure $5 \mathrm{a}$ contains all the values calculated with $c$ ranging from -.73 to .23 . Figures $5 \mathrm{~b}-5 \mathrm{e}$ represent successive magnifications about the origin.

\section{Step 4. Transformation of the Measure}

Given $\sigma$ and $\mathbf{m}$, iterate the transformation $\mathbf{T}_{\sigma}$ on the set of measures until an eigenvector $\mathbf{m}_{\sigma}$ is found having eigenvalue $K(\sigma)$. This means that $\mathbf{T}_{\sigma}\left(\mathbf{m}_{\sigma}\left(A_{i}\right)\right)=$ $K(\sigma) \mathbf{m}_{\sigma}\left(A_{i}\right) . \quad \mathbf{T}_{\sigma}$ is a linear transformation, and the iteration will converge to the largest eigenvector. Renormalization of the transformed measure after each application of $\mathbf{T}_{\sigma}$ prevents the norm from approaching infinity. If the eigenvalue equals one, then $\mathbf{m}_{\sigma}$ is a fixed point for $\mathbf{T}_{\sigma}$ and $\delta=\sigma$ is the Hausdorff dimension. In that case, the algorithm terminates. Otherwise, we must change the value of $\sigma$.

\section{Step 5. Newton's Method Determines a New Choice of $\sigma$}

If we view the eigenvalue $K(\sigma)$ found in Step 4 as a function of $\sigma$, then we are looking for $\sigma$ satisfying $K(\sigma)=1$. This is equivalent to finding the point where the function $K(\sigma)-1$ equals zero. Use Newton's method for finding roots of functions to get

$$
\sigma_{\text {new }}=\sigma+(1-K(\sigma)) / K^{\prime}(\sigma) .
$$

Choose a small number $\varepsilon$ and use Step 4 to compute $K(\sigma+\varepsilon)$. The derivative $K^{\prime}(\sigma)$ can then be approximated as

$$
K^{\prime}(\sigma) \approx(K(\sigma+\varepsilon)-K(\sigma)) / \varepsilon .
$$




\section{Step 6. Reset the Measure and Dimension}

Let $\mathbf{m}$ now be the eigenvector $\mathbf{m}_{\sigma}$ and $\sigma$ be the $\sigma_{\text {new }}$ found in Step 5. Go back to Step 4. The loop terminates when Step 4 results in an eigenvalue of 1 .

4. Discussion of the Results. This algorithm was implemented in the $C$ programming language on a VAX computer. The output is from a laser printer. Figures 5a-e illustrate the results. Since the Hausdorff dimension is always at least one, the graph is of $H(c)-1$ where $H(c)$ is the Hausdorff dimension of the Julia set of the $z \rightarrow z^{2}+c$.

This method works for any hyperbolic polynomial with connected Julia set; however, the algorithm requires the points on the Julia set to be kept in circular order. This is easy to do when $c$ has no imaginary part. We are currently working on a program to extend this technique to complex values of $c$ using analytic continuation. The Julia sets for complex value of $c$ look even more convoluted than for real values, as is illustrated in Figure 4.

Although the program produces a beautiful curve, there remain some parts of the theory not yet demonstrated. $H(c)$ depends upon the number of iterations $n$ used to form the partition. There are severe space limitations on how large $n$ can be, since the number of points is an exponential function of $n$. A computer experiment can be made to check how the Hausdorff dimension varies with the number of points in the Julia set. It is done as follows. Compute the Hausdorff dimension for a fixed number of iterations and then increase the number of iterations by one. Modify the algorithm in Step 3 by initializing the process using the Hausdorff dimension just computed for the smaller $n$ and its corresponding eigenmeasure. If this is done, then the process converges more rapidly, indicating that the previously computed measure and dimension for the smaller value of $n$ were already good approximations to the correct solutions. The dimension appears to converge rather rapidly for small values of $c$ and more slowly for large values. But we do not have a proof that this does converge, much less a handle on what the rate of convergence would be.

Acknowledgments. The author would like to thank Harvard University and the University of Colorado at Boulder for providing computing time for this project.

Department of Statistics and Computer Information Systems

Baruch College

New York, New York 10038

1. R. BowEN, "Hausdorff dimension of quasi-circles," Inst. Hautes Études Sci. Publ. Math., No. 50,1979 , pp. 11-25.

2. P. Blanchard, "Complex analytic dynamics on the Riemann sphere," Bull. Amer. Math. Soc. (N.S.), v. 11, 1984, pp. 85141.

3. A. DouAdy \& J. HubBard, "Iteration des polynomes quadratiques complexes," C.R. Acad. Sci. Paris Ser. I Math., v. 294, 1982, pp. 123-126.

4. B. Mandelbrot, The Fractal Geometry of Nature, Freeman, San Francisco, Calif., 1983.

5. B. MANDELBROT, "Fractal aspects of the iteration of $z \rightarrow \lambda z(1-z)$ for complex $\lambda$ and $z$," Nonlinear Dynamics (Internat. Conf., New York, 1979), Ann. New York Acad. Sci., vol. 357, New York Acad. Sci., New York, 1980, pp. 249259. 
6. V. A. NORTON, "Generation and display of geometric fractals in 3-D," Computer Graphics, v. 16, 1982, pp. 61-67.

7. E. OT T, "Strange attractors and chaotic motions of dynamical systems," Rev. Modern Phys., v. 53,1981 , pp. $655-671$.

8. C. A. Rogers, Hausdorff Measures, Cambridge University Press, Oxford, 1970.

9. D. RUELle, "Repellers for real analytic maps," Ergodic Theory Dynamical Systems, v. 2, 1982, pp. 99-108.

10. D. Sullivan, Seminar on Conformal and Hyperbolic Geometry, Inst. Hautes Études Sci. Seminar notes, 1982, pp. 1-92. 